\title{
Astronomical Observations of Comets and the Io Torus Using Fabry-Perot-Interferometry
}

\author{
Klaus Jockers \\ Max-Planck-Institut für Aeronomie, Postfach 20, D-37189 \\ Katlenburg-Lindau, Germany
}

\begin{abstract}
Fabry-Perot interferometers have been applied in a focal reducer to observe radiation from atoms and molecules in comets and in the Io torus. Tunable Fabry-Perot interferometers have been used as narrowband filters, and an etalon with fixed airgap in classical arrangement was employed for the measurement of Doppler shifts and for narrow-line photometry.
\end{abstract}

\section{Introduction}

Comets, as well as the neutral clouds and the ionized torus, associated with Jupiter's satellite Io, are extended objects which emit an emission line spectrum on a quasi-continuous background which in the comets is caused by sunlight scattered by the cometary dust particles and in case of Io originates from the light of bright nearby Jupiter. In contrast to most other astronomical objects, however, comets, as well as the Io torus, quickly change their appearance. The ions in the coma and in the tail of comets vary within tens of minutes. The Io torus depends on Jupiter's longitude and the position of Io. Its time of change is of the order of half an hour. Therefore, time adds another dimension to the observational problem and makes it four-dimensional. Depending on the scientific task we will select the most suited three-dimensional hyperplane of four-dimensional space. Useful observations have been made with a conventional long-slit spectrograph, i. e. by sacrificing one of the spatial dimensions. In the future we may perform interesting observations with the new devices for three-dimensional spectrography presented at this conference. This paper deals with the case, where the wavelength dimension has been reduced to one or two wavelengths for two-dimensional narrow-band imaging, or to a neighbourhood of one wavelength in order to derive Doppler speeds. Fabry-Perot interferometers are well suited for both of these tasks.

\section{Scientifle aim of the investigations of comets and the Io torus}

\subsection{Cometary ions}

The work presented in this study concerns investigation of cometary ions in the vicinity of the cometary nucleus, in particular $\mathrm{H}_{2} \mathrm{O}^{+}$and $\mathrm{CO}^{+}$, and to a lesser extent, $\mathrm{CO}_{2}^{+}$. These ions, which have emissions in the visible range of the spectrum, are generated directly from the most important parent substances 
$\mathrm{H}_{2} \mathrm{O}$ and $\mathrm{CO}_{2}$ (or perhaps $\mathrm{CO}$ ) by photoionization or charge exchange with the solar wind protons (Dissociative ionization produces $\mathrm{CO}^{+}$from $\mathrm{CO}_{2}$, see Huebner and Giguere, 1980). A summary of the important reactions in comets can be found in Schmidt and Wegmann (1980). If the water production of the comet is known, which can be derived from observations of the forbidden oxygen line at $630 \mathrm{~nm}$, observations of cometary ions in the cometary coma allow to verify the ionization reactions. In addition, the comet-solar wind interaction and the origin of tail rays and clouds in the inner coma can be studied from such observations and be compared with model calculations (e. g. Wegmann et al. 1987). Of particular interest is the question, how to explain the activity of comets at heliocentric distances where water does not sublimate anymore. There seems to exist a rare class of carbon-rich comets which show strong $\mathrm{CO}^{+}$tails at distances beyond $3 \mathrm{AU}$, like comet Morehouse $1908 \mathrm{III}$ and comet Humason 1962 VIII. Comet Schwassmann-Wachmann 1 (SW1), which has a nearly circular orbit slightly beyond Jupiter and is famous for its frequent outbursts, possibly also belongs to this group. $\mathrm{CO}^{+}$has first been detected in this comet by Cochran et al. (1980) and Larson (1980). The recent detections of neutral CO in the radio range by Senay and Jewitt (1994) and by Lellouch et al. (1994) indicate a $\mathrm{CO}$ production of this comet of $3 \times 10^{28} \mathrm{CO}$ molecules $\mathrm{s}^{-1}$. Comet $\mathrm{SW} 1$ produces at $\approx 6 \mathrm{AU}$ from the Sun a comparable number of $\mathrm{CO}$ molecules as comet Halley at the Giotto encounter, inside of Earth's orbit (Eberhardt et al. 1987).

\subsection{Neutrals and ions associated with Jupiter's satellite Io}

The Laplace resonance between the three inner Galilean moons causes stresses in Io, the innermost of them (Greenberg, 1982). This leads to heating in the interior of Io and to vulcanism on its surface. Some of the gas escapes Io and forms neutral clouds in Io's vicinity. When the material is ionized it is picked up by Jupiter's magnetic field and forms the Io torus, much like cometary ions are picked up by the solar wind magnetic field. Because of Jupiter's rapid rotation the torus is located close to the equator of Jupiter's tilted magnetic dipole and rotates nearly synchronously with Jupiter's rotation. The physics of the Io torus has recently be summarized by Thomas (1992). Topics of interest are the time variation of electron density to be deduced from the ratio of the two red [SII] lines, detection and investigation of the spatial distribution of oxygen in different ionization stages and of neutral and doubly ionized sulfur. So far, besides of readily excitable $\mathrm{Na}$ and $\mathrm{K}$, only various ionization stages of sulphur and oxygen have been observed in the visible spectral range.

\section{The instrument}

The instrumentation used for the present studies developed from the focal reducer of Hoher List Observatory (see Geyer et al. 1979). During the years it underwent considerable changes and improvements. The instrument development was done in cooperation with E. H. Geyer. The focal reducer is an all-lens design. The light collected by the telescope passes the Cassegrain focal plane and a field lens and is made parallel by a three-lens collimator. Presently there are two collimator units. One fits the $1 \mathrm{~m}$ telescope of Hoher List Observatory and its ESO twin. It is owned by Hoher List Observatory and has a focal ratio of 
f/14.5. The other is owned by the Max-Planck-Institute for Aeronomy and has a focal ratio of $\mathrm{f} / 8$. It has been used at the $1.26 \mathrm{~m}$ and $2.2 \mathrm{~m}$ telescopes of Calar Alto Observatory, at the ESO $2.2 \mathrm{~m}$ telescope and at the $2 \mathrm{~m}$ telescope of the Bulgarian National Observatory (BNAO). Both collimator units have provision for putting masks and slits into the Cassegrain focal plane and a stepping motor driven offset guider which allows to track objects with non-sidereal motion. The optics provides a useful field of $10^{\circ}$. The image of the telescope pupil (exit pupil) is located $125(150) \mathrm{mm}$ behind the collimator lens of the $\mathrm{f} / 8(\mathrm{f} / 14.5)$ system and has a diameter of $50 \mathrm{~mm}$. In the parallel beam we can put interference filters, grating prisms for long-slit and multi-slit spectroscopy (see Jockers et al. 1987, Debi Prasad et al. 1992a), an arrangement of Wollaston prisms for imaging polarimetry (Geyer et al. 1994), and several Fabry-Perot interferometers (FPIs). All these devices are mounted in separate units and can be arranged in a flexible way. There is also a guiding unit for use in the parallel beam. It deflects the green light with a dichroic mirror and uses it for direct guiding on the cometary nucleus or on the satellite Io. After the rays have passed the parallel beam section of the focal reducer they are imaged by a camera unit on a CCD with $576 \times 385$ pixel of $22 \mu \mathrm{m}$ square size. The camera unit is equipped with two spectroscopic lenses, one for the near UV and blue range from $360-500 \mathrm{~nm}$, and one for the visual range from $420-680 \mathrm{~nm}$. Both lenses have an aperture of $50 \mathrm{~mm}$ and a focal length of $140 \mathrm{~mm}$. A commercial, non-spectroscopic lens with $75 \mathrm{~mm}$ focal length is also available and has been used for the projects of Section 4.2.

Presently there exist two tunable FPIs (TFPIs) with piezoceramic spacers and one fixed airgap etalon. The fixed airgap FPI works from 410 to $700 \mathrm{~nm}$, has at $620 \mathrm{~nm}$ a free spectral range of $0.21 \mathrm{~nm}$ and a finesse of about 15 . One TFPI (UV/blue) works between $360-430 \mathrm{~nm}$ with a free spectral range of 17 $\mathrm{nm}$ at $404 \mathrm{~nm}$ and a finesse of about 12 . The other (visual) TFPI works from $410-700 \mathrm{~nm}$, has at $620 \mathrm{~nm}$ a free spectral range of $5.9 \mathrm{~nm}$ and a finesse of 16 .

\section{Results}

\subsection{Tunable Fabry-Perot interferometers}

Distribution of $\mathrm{CO}^{+}, \mathrm{CO}_{2}^{+}$and $\mathrm{OH}^{+}$in comet Halley 1986 III (ESO $1 \mathrm{~m}$ telescope) The brightest comet which so far has been observed with this equipment for an extended time period was comet Halley (Jockers et al. 1987). At that time, however, the detector was a two-stage proximity focus image intensifier with bialkali cathode. It was insensitive in the red spectral region but had a good efficiency in the UV up to $550 \mathrm{~nm}$. Unfortunately, blooming effects and afterglow of the intensifier, and nonlinear response of the photographic plates which were used to record the images prevented a photometric reduction of the images. With the UV/blue TFPI the comet was successfully imaged in the light of $\mathrm{CO}^{+}$at 400 and $425 \mathrm{~nm}$. Surprisingly, the best and cleanest images of the plasma coma were obtained at $367.4 \mathrm{~nm}$ in the Fox-Duffendack-Barker system of $\mathrm{CO}_{2}^{+}$. Images of $\mathrm{OH}^{+}$were taken at $358 \mathrm{~nm}$. Never since has it been possible to explore this extremely interesting spectral region in a comet. 
Distribution of $\mathrm{H}_{2} \mathrm{O}^{+}$in comet Austin $1990 \mathrm{~V}$ (Hoher List $1 \mathrm{~m}$ telescope) After the image intensifier had been replaced with a CCD, which has excellent red response, it was clear that the ion to be explored now was $\mathrm{H}_{2} \mathrm{O}^{+}$at $620 \mathrm{~nm}$. In this spectral range the dust emission is stronger so, in order to suppress the dust continuum, a higher resolution of the FPI was needed. This led to aquisition of the TFPI for the visual range. With the resolution of this TFPI the Fabry-Perot fringe is already noticable in the field of the instrument. The location of the fringe in the image is optimized at the telescope before the observations and verified afterwards. During data reduction, the calibration fringe is fitted by an Airy-function. To some extent it is also possible to correct for drifts of the TFPI. The drifts occur when during changing humidity conditions the dielectric reflective coatings of the FPI adsorb or release moisture, and the phase jump at reflection is changed. The water ions in comet Austin $1990 \mathrm{~V}$ were successfully observed (Bonev and Jockers, 1994). Because of limited space I cannot present the results in detail. In order to remove from the images the light scattered by the dust, which is the dominating emission close to the nucleus, the comet was imaged in the $\mathrm{H}_{2} \mathrm{O}^{+}$line and in a nearby continuum window. Realizing that only the line emission is modulated by the response of the FPI we can write two equations which describe the contribution of dust continuum and $\mathrm{H}_{2} \mathrm{O}^{+}$ line to each image. This system of equations can be solved for the line emission contribution. In this way the continuum can be completely removed. This approach is valid if the line width of the observed line is small as compared to the resolution of the FPI.

Distribution of $\mathrm{CO}^{+}$in Comet Schwassmann-Wachmann 1 (2 $m$ telescope of $B N A O$ ) In December $1992 \mathrm{CO}^{+}$was observed with the UV/blue TFPI in comet SW1 at the $2 \mathrm{~m}$ telescope of the Bulgarian National Observatory (Jockers, Chernova and Bonev, in preparation). After a whole night of integrating alternatingly in the $(3-0) \Pi_{3 / 2}$ subband of $\mathrm{CO}^{+}$at $402 \mathrm{~nm}$ and in a nearby continuum the emission is marginally detected. The production rate of $\mathrm{CO}^{+}$ is of the order of $10^{27} \mathrm{CO}^{+}$ions per second, $i$. e. about one tenth of the $\mathrm{CO}$ production of this comet. Because the solar irradiance at comet SW1 is only about $1 / 30$ of the irradiance at Earth even this high production rate is already at the detection limit. The $\mathrm{CO}^{+}$coma and tail of comet SW1 is likely to be very extended. Therefore part of the detection problem comes from the small field of the focal reducer. The derived $\mathrm{CO}^{+}$production rate may still be underestimated.

Red [SII] lines in the Io torus ( $2 \mathrm{~m}$ telescope of BNAO) Observations of the Io torus close to Jupiter present a real challenge. In order to minimize on one hand straylight from Jupiter, and on the other hand image Jupiter on the CCD for reference purposes, at the Cassegrain focal plane Jupiter is placed behind a black glass ( $1 \mathrm{~mm}$ of Schott glass NG1). The black glass has an angle of $15^{\circ}$ with the incident light. The image reflected off the black glass is collected in a guiding telescope for direct guiding on Jupiter. Two movable sliders on both sides of the black glass act as adjustable field stops and allow to block the light from the Galilean satellites, if necessary. A Lyot stop is put at the exit pupil of the collimator. The high resolution of the "visual" FPI effectively suppresses the quasicontinuum of the stray light from Jupiter and is 
well sufficient to separate the two [SII] lines at 671.6 and $673.0 \mathrm{~nm}$. An additional complication is introduced by the rotation of the torus with a speed of $74 \mathrm{~km}$ $\mathbf{s}^{-1}$. If the position of Jupiter on the CCD is known the line of sight velocity of the torus material can be calculated as a function of the pixel coordinates. Using the Airy-function fit to the optimized fringe of the TFPI (see above) and the known velocity field the response of the TFPI to the Doppler-shifted emission from the torus can be determined accurately as a function of the pixel coordinates. As it turns out, if Jupiter is at the field centre, the east ansa of the torus, approaching the observer, can be more effectively imaged. This is because the blue shift of the emission lines increases with increasing distance from field centre, in accordance with the blue shift of the FPI for larger angles of incidence. In order to effectively image the west ansa of the torus, Jupiter must be put to the edge of the CCD field.

\subsection{Etalon with fixed air gap}

Doppler velocity maps of comet Levy's (1990 XX) plasma tail (Hoher List $1 \mathrm{~m}$ telescope) Rauer and Jockers (1993) have determined column densities and the Doppler velocity field in comet Levy 1990 XX. Because of lack of space I refer to the publication for the details. In principle one could try to use a TFPI of high resolution to obtain a data cube. During the time needed to accumulate enough photons for the data cube the plama tail will change and impair comparison of the Doppler-shifted images (Schultz, 1993). Instead the fixed-gap FPI was put in the parallel beam and the velocity field obtained in the classical way by measuring the radius of the fringes. To get also the column density distribution images with and without FPI were taken alternatingly. Once each night an image through an interference filter of $3 \mathrm{~nm}$ full width at half maximum was taken in a dust continuum window centered at $642 \mathrm{~nm}$. This dust image was subtracted from the water ion images and from the interferograms. All images taken without FPI were saturated close to the nucleus which resulted in loss of the information in this area. As the study aimed at the determination of tail velocities loss of information close to the nucleus could be tolerated.

[OI] in comet Levy $1990 \mathrm{XX}$ (Hoher List $1 \mathrm{~m}$ telescope) While the cometary ions have outflow speeds between $20-100 \mathrm{~km} \mathrm{~s}^{-1}$ the neutrals move with speeds of about $1 \mathrm{~km} \mathrm{~s}^{-1}$. With the same setup as used for the determination of ion speeds, selecting a prefilter for the [OI] line at $630 \mathrm{~nm}$, we obtained narrow fringes of the cometary oxygen line, separated from the terrestrial air glow line because of the motion of the comet with respect to the earth (Debi-Prasad et al., 1992b). This allows to determine the extent of the [OI] coma around comets to large distances where the cometary line is already faint as compared to the air glow line.

\section{Conclusions}

FPIs can be used with advantage to observe comets and the Io torus. The new devices for three-dimensional spectroscopy presented at this conference, however, open new possibilities, and it seems worthwhile to examine their use for the observation of solar system objects in the future. 


\section{Discussion}

T. Herbst: Do you have observing plans of the Shoemaker-Levy 9 collision with Jupiter this summer?

$K$. Jockers: Yes. I would like to search for [OI] in this comet using the method described above. At the time of the observations comet Shoemaker-Levy 9 will, however, have zero geocentric velocity so we cannot separate the cometary line from the air glow line. Therefore I will not use FPIs and do only wide-band imaging.

\section{References}

Bonev, T., \& Jockers, K., 1994, Icarus 107, 335

Cochran, A. L., Barker, E. S., \& Cochran, W. D., 1980, AJ85, 474

Debi Prasad, C., Jockers, K., \& Geyer, E. H., 1992a, Icarus, 95, 211

Debi Prasad, C., Jockers, K., Rauer, H., \& Geyer, E. H., 1992b, Asteroids, Comets, Meteors, 1991 (Flagstaff), A. W. Harris and E. Bowell, Lunar and Planetary Inst., Houston, 477

Eberhardt, P., Krankowsky, D., Schulte, W., Dolder, U., Lämmerzahl, P., Berthelier, J. J.,, Woweries, J., Stubbemann, U., Hodges, R. R., Hoffman, J. H., \& Illiano, J. M., 1987, A\&A187, 481

Geyer, E. H., Jockers, K., \& Kiselev, N. N., 1994, A\&A, submitted

Geyer, E. H., Hoffmann, M., \& Nelles, B., 1979, A\&A, 80, 248

Greenberg, R., 1982, in Satellites of Jupiter, D. Morrison and M. Shapley Matthews, Tucson, U. of Arizona Press, 65

Jockers, K., Geyer, E. H., Rosenbauer, H., \& Hänel, A. 1987, A\&A, 187, 256

Huebner, W. F., \& Giguere, P. T., 1980, ApJ238, 753

Larson, S. M., 198, ApJ238, L47

Lellouch, E., Paubert, G., Biver, N., Bockelee-Morvan, D., Colom, P., Crovisier, J., Jorda, L., Rocher, P., \& Despois, D., 1994, IAU Circular 5995

Rauer, H., \& Jockers, K., 1993, Icarus 102, 117

Schmidt, H. U., \& Wegmann, R, 1980, Comp. Phys. Comm. 19, 309

Schultz, D., 1993, Fabry-Perot observations of comet Austin $1989 c_{1}=1990 \mathrm{~V}$, Ph. d. thesis, U. of Wisconsin, Madison

Senay, M. C., \& Jewitt, D. , 1994, IAU Circular 5929

Thomas, N., 1992, Surveys in Geophysics 13, 91

Wegmann, R., Schmidt, H. U., Huebner, W. F., \& Boice, D. C., 1987, A\&A, 187,339 